\title{
Relaxation time of the fermions in the magnetic field (II) - away from strong magnetic field limit -
}

\section{Tetsuya Onogi* and Aya Kagimura}

Department of Physics, Osaka University, Osaka560-0043, Japan

E-mail: kagimuradhet.phys.sci.osaka-u.ac.jp

The relaxation time for the fermions in the magnetic field is needed for estimating the chiral magnetic effect. However, it has been estimated only in the strong magnetic field. We discuss the relaxation time away from the strong magnetic field limit.

34th annual International Symposium on Lattice Field Theory

24-30 July 2016

University of Southampton, $U K$

\footnotetext{
${ }^{*}$ Speaker.
} 


\section{Introduction}

The electric conductivity of the semi-metals under the strong Magnetic Field with the Electric Field parallel to it offers an interesting physics. In this circumstance, it was shown that in the strong magnetic field limit, the electric current can be understood as a manifestation of chiral anomaly effect and is proportional to the $\mathrm{U}(1)$ chiral anomaly as

$$
J \propto E \cdot B .
$$

This phenomena was originally predicted by Argyres and Adams [W] and further studied by Nielsen and Ninomiya [ $[2]$. This phenomena is now called chiral magnetic effect and is now getting a renewed interest in the quark gluon plasma [3]. Recently experiments has started observing the

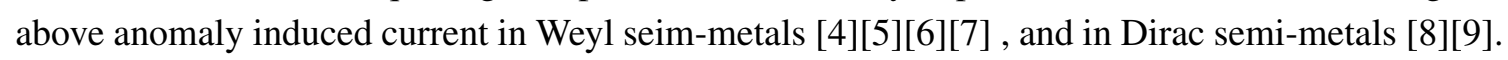
Since the experiment measures the magnetic field dependence of the conductivity for a wide range of 0 to 10 Tesla, it would be interesting to understand it even away from the strong magnetic field limit.

\section{Basics of the transport theory}

The mechanism of the chiral magnetic effect is the following. Let us take the direction of the magnetic field as the $z$ axis. Due to the strong magnetic effect, the electron states in the $x$ and $y$ directions form Landau levels. If one is interested in the low energy physics at the strong magnetic field limit, only the lowest Landau levels contribute to the physics so that the system effectively becomes the system of electrons in $1+1$ dimension. When electric field in the $z$ direction, the electrons receive a drift force and electric charge flows. Due to the scattering with impurities, phonons or other electrons the momentum of the electrons are flipped which makes the static current flow as a result of the balance between the drift and the scattering as shown in Fig.W

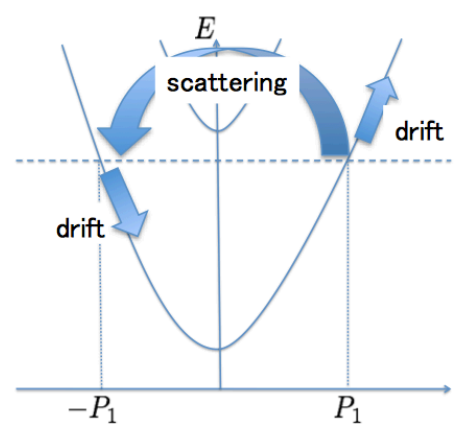

Figure 1: Drift from the electric field versus the scattering effect.

This means that the scattering time determines the size of the current. So far the scattering time is computed in the strong mangetic field regime where only the lowest Landau level contribute. In this report, we calculate the scattering time for weaker magnetic field regime where the second lowest Landau levels also contribute. For simplicity, we work at zero temperature and assume that 
the scattering with impurities is the main source. We also employ the low energy effective field theory to describe the electron near the Fermi level. Although we have to work for the relativistic fermion to describe the low energy behavior of the electrons in Weyl and Dirac semi-metals, in this exploratory study we employ non relativistic fermion as a toy model. The relativistic effect is study by another author [ए]].

Consider the probability distribution function $f\left(n, P_{y}, P_{z}, t\right)$ for the electrons at the $n$-th Landau level with momentum $P_{y}, P_{z}$ in the $y$ and $z$ directions. Here, we have taken the gauge for the vector potential corresponding to the magnetic field is given as

$$
A=(0, B x, 0),
$$

where $B$ is the magnitude of the magnetic field. In this gauge, the momentum $P_{y}$ is a good quantum number which is the labels to distinguish the degenerate states within the $n$-th Landau level. Applying a weak electric field

$$
E=(0,0, E)
$$

the Boltzmann equation is given as

$$
\frac{\partial}{\partial t} f\left(n, P_{y}, P_{z}, t\right)-e E \frac{\partial}{\partial P_{z}} f\left(n, P_{y}, P_{z}, t\right)=\left(\frac{\partial}{\partial t} f\left(n, P_{y}, P_{z}, t\right)\right)_{\text {collision }},
$$

where the second term on the left hand side is the drift term and the right hand side is the collision term. The collision term is defined as

$$
\begin{aligned}
& \left(\frac{\partial}{\partial t} f\left(n, P_{y}, P_{z}, t\right)\right)_{\text {collision }} \\
= & -\sum_{n^{\prime}} \int \frac{d^{2} P^{\prime}}{(2 \pi)^{2}} f\left(n, P_{y}, P_{z}, t\right) W\left(n, P_{y}, P_{z} \rightarrow n^{\prime}, P_{y}^{\prime}, P_{z}^{\prime}\right)\left(1-f\left(n^{\prime}, P_{y}^{\prime}, P_{z}^{\prime}, t\right)\right) \\
& +\sum_{n^{\prime}} \int \frac{d^{2} P^{\prime}}{(2 \pi)^{2}} f\left(n^{\prime}, P_{y}^{\prime}, P_{z}^{\prime}, t\right) W\left(n^{\prime}, P_{y}^{\prime}, P_{z}^{\prime} \rightarrow n, P_{y}, P_{z}\right)\left(1-f\left(n, P_{y}, P_{z}, t\right)\right),
\end{aligned}
$$

where $W$ is the transition probability per unit time.

We now consider the case where the electric field is very weak and can be treated perturbatively. Then the probability distribution function can be desrcibed as

$$
f\left(n, P_{y}, P_{z}, t\right)=f_{0}\left(\varepsilon\left(n, P_{z}\right)\right)+\delta f\left(n, P_{y}, P_{z}, t\right),
$$

where $f_{0}$ is the probability distribution function in equilibrium for the Landau level with no electric field and $\delta f$ is a small deviation of $O(e E) . \varepsilon\left(n, P_{z}\right)$ is the energy of the electron at the $n$-th Landau level and momentum $P_{z}$. (Note that $P_{y}$ does not contribute to the energy. )

Assuming small deviation from the equilibrium and taking only linear term in $\delta f$, the defining equation for the collision term reduces to

$$
\begin{aligned}
& \left(\frac{\partial}{\partial t} f\left(n, P_{y}, P_{z}, t\right)\right)_{\text {collision }} \\
= & -\sum_{n^{\prime}} \int \frac{d^{2} P^{\prime}}{(2 \pi)^{2}} W\left(n, P_{y}, P_{z} \rightarrow n^{\prime}, P_{y}^{\prime}, P_{z}^{\prime}\right)\left(\delta f\left(n, P_{y}, P_{z}, t\right)-\delta f\left(n^{\prime}, P_{y}^{\prime}, P_{z}^{\prime}, t\right)\right) .
\end{aligned}
$$


To solve the Boltzmann equation, one often makes the relaxation time approximation

$$
\left(\frac{\partial}{\partial t} f\left(n, P_{y}, P_{z}, t\right)\right)_{\text {collision }} \approx-\frac{1}{\tau\left(n, P_{y}, P_{z}\right)} \delta f\left(n, P_{y}, P_{z}\right) .
$$

Substituting Eq. ([2.6) to Eq.([2.3]), the static solution of the Boltzmann equation is given as

$$
\delta f\left(n, P_{y}, P_{z}\right)=\tau\left(n, P_{y}, P_{z}\right) e E \frac{\partial}{\partial P_{z}} f_{0}\left(\varepsilon\left(n, P_{z}\right)\right)=\tau\left(n, P_{y}, P_{z}\right) e E \frac{\partial \varepsilon\left(n, P_{z}\right)}{\partial P_{z}} f_{0}^{\prime}(\varepsilon)
$$

Substituting Eq.([2.7) and Eq.([2.8) into Eq.([2., and using the energy conservation in Fermi's Golden rule, one obtains

$$
\begin{aligned}
& \frac{\partial \varepsilon\left(n, P_{z}\right)}{\partial P_{z}} \\
= & \sum_{n^{\prime}} \int \frac{d^{2} P^{\prime}}{(2 \pi)^{2}} W\left(n, P_{y}, P_{z} \rightarrow n^{\prime}, P_{y}^{\prime}, P_{z}^{\prime}\right)\left(\tau\left(n, P_{y}, P_{z}\right) \frac{\partial \varepsilon\left(n, P_{z}\right)}{\partial P_{z}}-\tau\left(n^{\prime}, P_{y}^{\prime}, P_{z}^{\prime}\right) \frac{\partial \varepsilon\left(n, P_{z}^{\prime}\right)}{\partial P_{z}^{\prime}}\right)
\end{aligned}
$$

from which one can determine the scattering time $\tau$. In the case of electrons under the magnetic field, the energy is given as

$$
\varepsilon\left(n, P_{y}, P_{z}\right)=\frac{e B}{m}(n+1 / 2)+\frac{P_{z}^{2}}{2 m},
$$

where $m$ is the mass of the electron. Eq.(ㅁ.g) is further simplified as

$$
P_{z}=\sum_{n^{\prime}} \int \frac{d^{2} P^{\prime}}{(2 \pi)^{2}} W\left(n, P_{y}, P_{z} \rightarrow n^{\prime}, P_{y}^{\prime}, P_{z}^{\prime}\right)\left(\tau\left(n, P_{y}, P_{z}\right) P_{z}-\tau\left(n^{\prime}, P_{y}^{\prime}, P_{z}^{\prime}\right) P_{z}^{\prime}\right)
$$

In Ref.[四], they computed the scattering time for non relativistic fermion system in the strong coupling regime where only the lowest Landau level $(n=0)$ contributes.

\section{Coupled equations for the relaxation time}

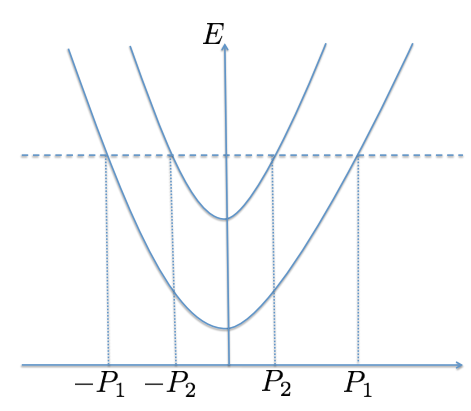

Figure 2: Momenta for $n=0$ and $n=1$ Landau levels

We extend the work by Adams and Argyres in Ref.[四] and compute the scattering time for weaker magnetic field for which $n=1$ Landau levels also contribute. In Fig.】, the momenta in the $z$ direction at the Fermi level is denoted as $\pm P_{1}$ and $\pm P_{2}$, where the former mometa are at the $n=0$ Landau level and the latter momenta are at the $n=1$ Landau level. 


\section{Solutions}

We assume that the scattering time is independent of $P_{y}$. At zero temperature, only four set of states at the Fermi level contributes (labeled by $I=1,2,3,4$ ) at the Fermi level contribute. From Fermi's golden rule, the energy is conserved before and after the transition so that the probability can be written as

$$
W\left(n, P_{y}, P_{z} \rightarrow, n^{\prime}, P_{y}^{\prime}, P_{z}^{\prime}\right) \equiv 2 \pi \delta\left(\varepsilon\left(n, P_{z}\right)-\varepsilon\left(n^{\prime}, P_{z}^{\prime}\right)\right) \bar{W}\left(n, P_{y}, P_{z} \rightarrow, n^{\prime}, P_{y}^{\prime}, P_{z}^{\prime}\right) .
$$

Defining $w_{I J}$ as

$$
w_{I J}=\int \frac{d P_{y}^{\prime}}{2 \pi} \bar{W}\left(n_{I}, P_{y}, P_{I} \rightarrow, n_{J}, P_{y}^{\prime}, P_{J}\right)
$$

and integrating over $P^{p}$ rime $_{y}, P_{z}^{\prime}$, we obtain from Eq.([D])

$$
P_{I}=\sum_{J=1}^{4} w_{I J}\left(\tau_{I} P_{I}-\tau_{J} P_{J}\right) \frac{\mu}{\left|P_{J}\right|} \cdot \quad(I=1,2,3,4)
$$

Since the probability distributions $f$ and $f_{0}$ are normalized to unity, their difference satisfies

$$
\sum_{n} \int \frac{d^{2} P}{(2 \pi)^{2}} \delta f\left(n, P_{y}, P_{z}\right)=0
$$

from which one obtains using Eq.(‥8)

$$
\tau_{1}+\tau_{2}-\tau_{3}-\tau_{4}=0
$$

From a consideration of symmetries, one expects

$$
\begin{aligned}
& w_{I J}=w_{J I} \quad(I, J=1,2,3,4) \\
& w_{12}=w_{43}, w_{13}=w_{42}
\end{aligned}
$$

Combining Eq.(4.31) and Eq.(4.5), we find that the solution is given as

$$
\begin{aligned}
\tau_{1} & =\tau_{4}=\frac{1}{\mu} \frac{\left(w_{23} P_{1}+w_{12} P_{2}\right) P_{1} P_{2}}{2\left(w_{12} w_{13}+w_{14} 23\right) P_{1} P_{2}+\left(w_{12}+w_{13}\right)\left(w_{23} P_{1}^{2}+w_{14} P_{2}^{2}\right)}, \\
\tau_{2} & =\tau_{3}=\frac{1}{\mu} \frac{\left(w_{12} P_{1}+w_{14} P_{2}\right) P_{1} P_{2}}{2\left(w_{12} w_{13}+w_{14} w_{23}\right) P_{1} P_{2}+\left(w_{12}+w_{13}\right)\left(w_{23} P_{1}^{2}+w_{14} P_{2}^{2}\right)} .
\end{aligned}
$$

Note that the result by Argyres and Adams

$$
\tau_{1}=\tau_{4}=\frac{P_{1}}{2 \mu w_{14}} .
$$

for the strong magnetic field regime can be reproduced by artificially taking the limit $w_{12}, w_{13} \rightarrow 0$.

Starting from the strong magnetic field regime, let us consider what happens as we make the magnetic field weaker. While the system is in the strong magnetic field regime, the scattering time obeys Eq.(4.10) and makes a smooth change since $w_{14}$ can depend on the magnetic field. After the system is in the weaker magnetic field regime, the scattering time obeys Eq.(4.8). 
At the border of the strong and weaker magnetic field regimes where the $n=1$ level just touches the Fermi level, one finds $P_{2}=0$ and $w_{23}=\infty$. In this case one finds that

$$
\tau_{1}=\tau_{2}=0
$$

This means that as $B$ becomes small and when $n=1$ Landau level starts to contribute, a jump of scattering time (and current) can occur, which could be an interesting sigmal for chiral magnetic effect.

\section{Summary and discussion}

We studied the relaxation time for the chiral magnetic effect away from the strong magnetic limit. We derived the coupled equations for the relaxation time starting from the Boltzmann equation in the relaxation time approximation for the weaker magnetic field regime where both of the Landau levels with $n=0$ and $n=1$ contribute. Combining with the normalization condition for the probability distribution function which also give another equation for the relaxation times, we obtained solution to the coupled equation.

As a future plan, one needs to extend the present toy model calculation to the real calculation for relativistic fermion in order to understand the physics of Weyl or Dirac semi-metals. Also, the evaluation for the scattering time for a quantitative prediction of the conductivity is needed.

\section{References}

[1] P. N. Argyres and E. N. Adams, "Longitudinal magnetoresistance in the quantum limit", Phys. Rev. 104 (Nov, 1956) 900-908.

[2] H. B. Nielsen and M. Ninomiya, "ADLER-BELL-JACKIW ANOMALY AND WEYL FERMIONS IN CRYSTAL", Phys. Lett. B130(1983) 389.

[3] K. Fukushima, D. E. Kharzeev and H. J. Warringa, "The Chiral Magnetic Effect", Phys. Rev. D 78(Oct., 2008) 074033, [0808.3382]].

[4] X. Wan, A. M. Turner, A. Vishwanath and S. Y. Savrasov, "Topological semimetal and fermi-arc surface states in the electronic structure of pyrochlore iridates", Phys. Rev. B 83 (May, 2011) 205101.

[5] Q. Li, D. E. Kharzeev, C. Zhang, Y. Huang, I. Pletikosic, A. V. Fedorov et al., "Observation of the chiral magnetic effect in ZrTe5", Nature Phys. 12 (2016) 550-554, [14 42. 6543]].

[6] X. Huang, L. Zhao, Y. Long, P. Wang, D. Chen, Z. Yang et al., "Observation of the chiral-anomaly-induced negative magnetoresistance in 3d weyl semimetal taas", Phys. Rev. X 5 (Aug, $2015) 031023$.

[7] C. Zhang et al., "Observation of the Adler-Bell-Jackiw chiral anomaly in a Weyl semimetal", 1503.02630 .

[8] C. Zhang, E. Zhang, Y. Liu, Z.-G. Chen, S. Liang, J. Cao et al., "Detection of chiral anomaly and valley transport in Dirac semimetals", ArXiv e-prints (Apr., 2015), [1504.07698].

[9] J. Xiong, S. K. Kushwaha, T. Liang, J. W. Krizan, W. Wang, R. J. Cava et al., "Signature of the chiral anomaly in a Dirac semimetal: a current plume steered by a magnetic field", ArXiv e-prints (Mar., 2015), [11503.08179].

[10] Talk by Aya Kagimura, in these proceedings. 


\section{Acknowledgements}

This work is supported in part by the Grand-in-Aid of the Japanese Ministry of Education No. 26400248(T.O.). 